\title{
DETERMINATION OF OPTIMAL INSTRUCTIONAL TREATMENT IN ATI OF TWO APTITUDE DIMENSIONS AND MULTIPLE TREATMENTS ${ }^{1}$
}

\author{
HIROSHI NAMIKI AND JUNKO HAYASHI ${ }^{2}$ \\ Department of Educational Psychology, Keio University
}

\begin{abstract}
Under four types of instructional treatments, Text-Numerical, Text-Graphical, Program-Numerical and Program-Graphical, junior high school pupils were presented a learning task of understanding a formula to summate arithmetic progression. Aptitude treatment interactions were examined by taking two aptitude informations into consideration simultaneously, and optimal treatments were determined in the three-dimensional space of two aptitudes and one payoff. Significant interaction of general intelligence $\times$ GAT $\times$ Text-Program, for example, was identified by multiple regression analysis. The plane, whose co-ordinates are general intelligence and GAT, was divided into three regions of optimal treatments, Text-Numerical, Text-Graphical and ProgramNumerical. Payoffs of four types of treatments and optimized treatments were compared, and several other points in ATI research were discussed.
\end{abstract}

Aptitude-treatment interaction (ATI) study purports to design instructional methods which can be adapted to individual differences, or in short, optimized instructional treatment, through the inspection of interactional patterns among learners' aptitudes, structure of learning task and instructional treatment (Azuma, 1968; Cronbach, 1967). The practical importance of ATI study lies in its pos-

1 Part of this paper was presented in the proceedings of the 40 th annual convention of Japanese Psychological Association.

2 The authors wish to express their gratitude to Mr. Takeshi Shibata, Miss Sachiko Hayashi and Miss Yukiko Ishimura for their assistance in the accomplishment of experimental program. The authors also would like to express their appreciation to Professor Reiji Konno of Meiji University for his helpful suggestions on mathematical problems. The authors are also indebted to Mr. Takashi Naito for computer programming and calculation.

Request for reprints should be sent to Hiroshi Namiki, Department of Education, Keio University, Mita, Minato-ku, Tokyo 108, Japan. sible solution of the drop-out problem which is particularly urgent in our country. Nowadays, the difficulty level of school curricula is increasing, and the ability range of learners is widening with popularization of education. Thus, increasing numbers of drop-out pupils will result inevitably unless some substantial innovations in educational methods are made available. ATI study could be duly expected to contribute to these innovations by providing a basic knowledge of the adaptation of instructional treatments to wide-ranged individual differences.

Besides the practical aspect of its importance, ATI study has relevance to more theoretical and methodological problems of behavioral science in general. Recently Cronbach (1975) has persuasively reiterated the significance of ATI approach, or interactionistic viewpoint, by taking many adequate illustrations of interaction from diverse research areas. In spite of the widely recognized significance of ATI study, Cronbach and Snow (1969) had to conclude in their extensive 
review that progress toward the goal of identifying and understanding ATI had been slight.

In relation to what the present investigation pursues, we discuss several points on ATI study in turn.

\section{Number of Aptitude Dimensions and Treatments}

With several exceptions almost all of ATI experiments reported hitherto have taken only one aptitude dimension into consideration. The experiment by Koran, Snow, and McDonald (1971) was one of the exceptional cases. They treated simultaneously two ability dimensions, Film Memory and Hidden Figures Part I, and determined optimal instruction in terms of the intersection of two regression planes in three-dimensional space. To adapt appropriate instructional methods to individual differences necessitates a knowledge of the many facets of the learner's personality. The number of aptitudes considered, therefore, should be necessarily increased as the ATI study advances.

The present study treats simultaneously two aptitude dimensions, intelligence and anxiety, both of which have been widely acknowledged as determinants of learning behavior.

Regarding the number of treatments, the present study examined four instructional treatments by combining two factors of instruction having two levels each. Taking into consideration multiple treatment as well as two aptitude dimensions in ATI would be the due course of research in heightening the generality of ATI study.

The experiment by Koran, et al. mentioned above included two aptitude dimensions, but the treatments also numbered two. In this case, the solution of optimal treatment is comparatively simple and determined by bisecting the plane of two aptitude dimensions. In the case of the present study and another one by the present authors (Hayashi \& Namiki, 1975; Namiki, Hayashi, \& Kazama, 1977), determination of optimal treatments is rather complicated and, for example, the original form of the Johnson-Neyman technique (Potthoff, 1964) is of no use in specifying the region of significance.

\section{Determination of Optimal Treatment}

Generally with regard to two aptitude dimensions $\mathrm{x}$ and $\mathrm{y}$, and posttest score or payoff dimension $z$, the optimal treatment among two or more instructional treatments can be determined in the following manner.

Firstly, we obtain regression planes $z^{\prime}$ of payoff dimension $\mathrm{z}$ onto $\mathrm{x}$ and $\mathrm{y}$ :

$$
\mathbf{z}^{\prime}=\mathrm{a}_{i} \mathrm{x}+\mathrm{b}_{i} \mathrm{y}+\mathrm{c}_{i} \quad(i=1, \ldots \mathrm{k}),
$$

where suffix $i$ stands for an instructional treatment, and $\mathrm{k}$ represents the number of treatments. These $k$ regression planes cross each other, and form ${ }_{k} \mathrm{C}_{2}$ cross-lines in the three-dimensional space of $x, y$ and $z$. Then we can find orthogonal projections of these cross-lines on the xy plane. For example, with regard to regression planes 1 and 2, the orthogonal projection of the cross-line onto the xy plane can be obtained by eliminating $z^{\prime}$ from the two equations of these regression planes:

$$
\left(a_{1}-a_{2}\right) x+\left(b_{1}-b_{2}\right) y+\left(c_{1}-c_{2}\right)=0 .
$$

Lines on the xy plane, formed by projection, divide the plane into several regions $s_{j}(j=1,2, \ldots)$. If we evaluate the relative position of $\mathrm{k}$ regression planes along the $\mathrm{z}$ dimension upon a specific region $\mathrm{s}_{j}$, and decide which one of these regression planes takes the maximum $\mathrm{z}$ value, then we can obtain the optimal instructional treatment that corresponds uniquely to the specific region $s_{j}$. Therefore, we specify an arbitrary point $\left(\mathrm{x}_{\mathrm{s}_{j}}, \mathrm{y}_{\mathrm{s}_{j}}\right)$ in a region $\mathrm{s}_{j}$, and calculate $\mathrm{z}$ values for each $\mathrm{k}$ regression plane (designated $\mathrm{z}_{\mathrm{s}_{j}}$ ) at that point:

$$
\mathbf{z}_{\mathrm{s}_{j}}^{\prime}=\mathrm{a}_{i} \mathrm{x}_{\mathrm{s}_{j}}+\mathrm{b}_{i} \mathrm{y}_{\mathrm{s}_{j}}+\mathrm{c}_{i} .
$$

Then we decide the largest $z_{s_{j}}$ among the $\mathrm{k}$ values. Evaluating maximum $\mathrm{z}$ 
values in every region $\mathrm{s}_{j}$ on the $\mathrm{xy}$ plane in the same manner, we can obtain the polyhedron of optimal instructional treatment in a three-dimensional space as an orthogonal projection onto the xy plane.

\section{Variety of Aptitudes}

In ATI study in general, the aptitude dimension should meet the following two requirements. First, the aptitude variable should produce a differential slope of regression corresponding to each treatment. In the present state of ATI study, selection of aptitude dimension is empirical or haphazard rather than theoretical, despite the opinions of some authors (e.g. Rhetts, 1974) who criticize it from the methodological point of view. The difficulty lies in the fact that substantial methods are rarely available for either task or treatment analysis.

Secondly, aptitude variables should have some degree of generality. If aptitude variables are too task-specific, the outcomes of ATI study may be futile both theoretically and practically (Namiki, 1977; Tobias, 1973). On the other hand, if the aptitude variable is truly general, any treatment tends to yield a positive correlation with the aptitude dimension (Gronbach, 1967). Recently, however, Gronbach and Snow (1969) and Cronbach (1975) recommended the adoption of general intelligence in ATI study. This antinomy, viz. generality-specificity problem, is intrinsic to ATI study, and its solution must await further experimentation.

As an attempt at the solution of this problem, the present study, for use as variables of general aptitude, selected general intelligence and general anxiety. For specific aptitude, pretest score and State-Anxiety were chosen. These aptitude informations were expected to show interactions with the treatment factors of the present study. The text-program format variable, for example, was examined as one of several independent variables. Of course, our primary concern was with its possible interaction with the aptitude dimensions. The main effect of the text-program format variable and that of aptitude information are considered of less significance in the present paper.

\section{Summary of the Purpose}

The main purpose of the present investigation can be summarized as follows:

1) To establish a rule for determining optimal instructional treatment when two aptitude dimensions are simultaneously taken into consideration with regard to the multiple treatment of four instructional methods.

2) To identify a number of ATI effects ranging from first-order to higher-order interaction.

3) To compare payoffs of optimized treatments using information based upon two and single aptitude dimensions.

4) To check the reliability and practical utility of the obtained placement method by using a new sample of subjects.

5) To examine the generality-specificity problem of aptitude information in ATI study.

\section{Method}

\section{Experimental Factors and Variables}

The present study examined two experimental factors: Format of material and type of representation. The former makes a comparison between traditional textbook format and programmed book format; and the latter, between a numerical representation using mainly numerical expressions and a graphical representation in which graphs are used to facilitate the intuitive grasp of the learner. The following four instructional treatment conditions were arranged by combining these two factors : (1) Text format and numerical representation (T-Num Gr.), (2) text format and graphical representation (T-Gra Gr.), (3) program format and numerical representation 
(P-Num Gr.), (4) program format and graphical representation (P-Gra Gr.). Since subjects had no preliminary knowledge of the learning task at all, it was unnecessary to form a control group without instruction.

Besides these instructional treatment factors, the following aptitude informations were included as independent variables: Standard score of general intelligence (ISS), pretest score, grade point of mathematics, General Anxiety Test (GAT) score and State-Anxiety (A-State) score. General intelligence was measured by a group test named Todai A-type. The pretest, consisting of 8 items, presented subjects with simple arithmetic progressions and required them to fill in the last term of the progression. Grade point and general intelligence score were available from school records. GAT was composed of 100 items, and A-State was measured by a test of 24 items developed by the present authors following Spielberger's method (1966).

Dependent variables were two measures of payoff, i.e. immediate and retention test scores. The immediate test consisted of 6 items. Two of these items required subjects to reproduce formulas of the general term and the summation of an arithmetic progression, respectively; the remaining four items required subjects to apply these formulas to numerical examples. The retention test administered a week later was identical with the immediate test. These items were scored one point per correct response.

\section{Learning Task}

As the learning task the present study selected understanding and application of formula for the summation of an arithmetic progression. Gagné and Brown (1961) have used a similar task in their experiment, and the pertinence of this kind of task to experimental settings in education has been pointed out by Cronbach (1966) in his discussion of the discovery method. Although tasks of this difficulty level are currently only included in high school curriculum, the subjects of the present experiment taken from the junior high school population, demonstrated an ability to perform these same tasks.
Four kinds of learning materials were bound in booklet form corresponding to each instructional treatment condition. Careful consideration was given to ensure that the amount of information transmitted to learners was equal across the four conditions, though the format and type of instruction varied.

The program format was comprised of 28 frames and one frame was printed on each page. Following a requirement of a construct response to each frame, subjects were presented with the correct answer on the following page. An example of a frame in program format and numerical representation is shown in Fig. 1. In the graphical representation, for example, the common number 16 , obtained by summing the paired terms of Fig. 1, was expressed as the sum of two straight line segments.

A total of about 350 subjects participated in this experiment. They were second grade pupils in a junior high school located in an urban area of the Tokyo Metropolis. Since school records were deficient for some pupils,

It is interesting to note that the summation of each upper and lower terms equals the number 16. Utilizing this fact, summation of arithmetic progression can be calculated easily.

Summation of original progression $S$ and that of reversed order progression $S$ are naturally the same number because terms of each progression, even if the order of terms is reversed, are originally the same.

That is :

$$
\begin{aligned}
& S=2+4+6+8+10+12+14 \\
& S=14+12+10+8+6+4+2
\end{aligned}
$$

Placing the equations directly above one another add up each of the two member columns, to produce identical sums of 16 . Similarly, add the left halves of the equations and write your answer in the blank square provided.

$$
\begin{array}{r}
S=2+4+6+8+10+12+14 \\
+S=14+12+10+8+6+4+2 \\
\square=16+16+16+16+16+16+16
\end{array}
$$

FIG. 1. A sample frame of program format (P-Num condition). 
TABLE 1

Means and standard deviations of aptitude informations and payoffs in each instructional treatment group

\begin{tabular}{|c|c|c|c|c|c|c|c|c|c|c|}
\hline & & & \multicolumn{2}{|c|}{ T-Num } & \multicolumn{2}{|c|}{ T-Gra } & \multicolumn{2}{|c|}{ P-Num } & \multicolumn{2}{|c|}{ T-Gra } \\
\hline & & & $\mathcal{N}=70$ & $\mathcal{N}=56$ & $\mathcal{N}=70$ & $\mathcal{N}=56$ & $\mathcal{N}=70$ & $\mathcal{N}=56$ & $\mathcal{N}=70$ & $\mathcal{N}=56$ \\
\hline \multirow{10}{*}{$\begin{array}{l}\text { Aptitude } \\
\text { information }\end{array}$} & ISS & $M$ & 53.69 & 54.23 & 55.39 & 55.30 & 55.80 & 56.50 & 55.13 & 55.14 \\
\hline & & $S D$ & 7.89 & 7.46 & 7.45 & 7.76 & 8.96 & 8.04 & 9.19 & 8.87 \\
\hline & Mathematics & $M$ & 3.10 & 3.14 & 3.04 & 3.11 & 3.20 & 3.20 & 2.97 & 2.95 \\
\hline & & $S D$ & 0.90 & 0.88 & 0.94 & 0.97 & 0.93 & 0.94 & 1.06 & 1.07 \\
\hline & Pretest & $M$ & 7.79 & - & 7.60 & - & 7.57 & - & 7.64 & - \\
\hline & & $S D$ & 0.56 & - & 1.22 & - & 0.71 & - & 0.72 & - \\
\hline & GAT & $M$ & - & 49.64 & - & 50.32 & - & 51.02 & - & 49.13 \\
\hline & & $S D$ & - & 10.24 & - & 10.63 & - & 10.67 & - & 11.11 \\
\hline & A-State & $M$ & - & 56.27 & - & 56.32 & - & 56.95 & - & 55.05 \\
\hline & & $S D$ & - & 10.29 & - & 9.84 & - & 8.80 & - & 10.14 \\
\hline \multirow{4}{*}{ Payoff } & Immediate test & $M$ & 2.82 & 2.95 & 3.17 & 3.38 & 2.26 & 2.29 & 2.17 & 2.15 \\
\hline & & $S D$ & 2.35 & 2.30 & 2.43 & 2.44 & 2.05 & 2.05 & 2.19 & 2.15 \\
\hline & Retention test & $M$ & - & 1.60 & - & 1.96 & - & 1.57 & - & 1.19 \\
\hline & & $S D$ & - & 2.14 & - & 2.25 & - & 1.98 & - & 1.97 \\
\hline
\end{tabular}

and, during the second session of the experiment, a considerable number of pupils were absent due to influenza, the subjects analysed numbered 280 (70 subjects per group), or 224 (56 subjects per group). As a rule, the larger sample was used except when variables obtained in the second session were analysed. The second grade of this school consisted of eight classes, and each of the four instructional treatment groups was composed by combining two classes randomly. For the convenience of statistical calculation, four groups were reduced to the same size by discarding several subjects randomly. The resulting four groups were completely matched in general intelligence and other aptitude variables (Table 1).

\section{Procedure}

Administration of the present experiment required two sessions. In the first session, the pretest was administered with a $3 \mathrm{~min}$ time limit. Following the pretest, subjects were given the booklet of learning materials individually, and studied the task for $30 \mathrm{~min}$. To arouse motivation, subjects were given previous notice of the test which was to be administered after the learning period. Program format groups were allowed to reread the program if necessary. After the learning period, subjects took the immediate test for $10 \mathrm{~min}$. In the second session a week later, GAT was administered within $15 \mathrm{~min}$, and the retention test was given a $10 \mathrm{~min}$ time limit. Finally, State-Anxiety about this test situation was measured for $5 \mathrm{~min}$.

\section{Results and Discussion}

Tests of Interaction and Main Effect by Means of Multiple Regression Analysis

Analyses of data were performed mainly by multiple regression analysis usually recommended for ATI research (Cohen, 1968; Cronbach \& Snow, 1969; Kerlinger \& Pedhazur, 1973; Overall \& Spiegel, 1969). The succeeding analyses followed Method 3, that is, the step-down analysis described by Overall and Spiegel (1969). This method is recommended when some variables in the multiple regression equation are substantially intercorrelated as is the case with the present 
study. In dealing with such cases, this method permits an estimation of the contribution of each effect, adjusted for those preceding it in the equation, thus minimizing the possibility that the contribution of interaction terms, which is a matter of primary concern to us, might be underestimated. In using this method, however, the priority in the ordering of variables has a critical importance in estimating the relative contribution of each variable. Therefore, the significance of interaction terms was tested in several specified sequences outlined below.

In testing the significance of ATI effects with two instructional treatment factors and two aptitude informations, the full model was composed of the main effect terms of the following variables and the interaction terms from first-order up to third-order obtained by combining them. The variables considered were text-program and numerical-graphical, each as an instructional factor; ISS (or grade point of mathematics) and GAT score (or AState score), each as aptitude information. The pretest score was not included in this analysis because it showed a ceiling effect. Two instructional factors were treated as binary vectors by using effects coding (Kerlinger \& Pedhazur, 1973). Other aptitude variables and dependent variables were treated as continuous vectors. Dependent variables of these equations were either the immediate test score or the retention test score. As for the ATI effect with single aptitude information, the reduced models used included two instructional factors and the aptitude information plus their interaction terms from firstorder to second-order. Mention will be made of the results of these analyses in each following section.

Determination of Optimal Instructional Treatment in the Case of Tro Aptitude Dimensions and Multiple Treatments

Regarding the ATI effects of two instructional factors, ISS and GAT, when four main effect terms were given priority in the ordering, and when first-order to higher-order interaction terms followed the main effect terms, no interaction terms reached the significance level. But when the sequence was reversed, and higherorder interaction terms came first followed by lower-order interaction terms, and finally the main effects, the ATI of T-P $\times$ ISS $\times$ GAT was significant. This significant interaction partly supports the reliability of the obtained optimal solution as shown below since it includes only one instructional factor. The summary table of this multiple regression analysis is shown in Table 2.

Following the solution rule mentioned above, optimal treatment with ISS and GAT provided was so obtained as to divide the plane of two aptitude dimensions into three regions of optimal treatment (Fig. 2). For learners with high intelligence and high anxiety, T-Gra treatment is optimal; for those with low intelligence and low anxiety, T-Num treatment; and for low intelligence and high anxiety, P-Num treatment. No subjects in the present sample, however, fell into the last region; thus, it appeared only by extrapolation of the regression plane.

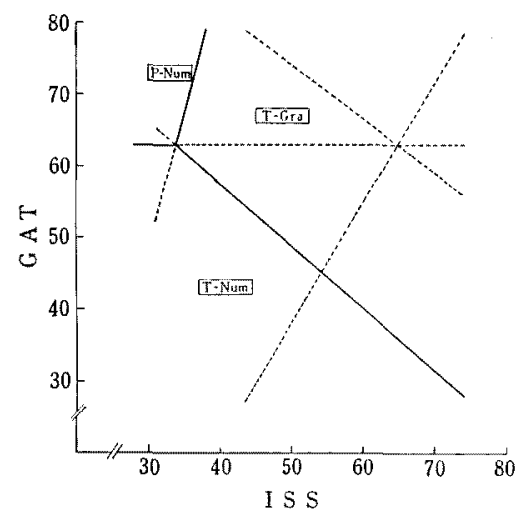

FIG. 2. Regions of optimal treatment when ISS and GAT are used as aptitude. Dotted lines are also orthogonal projections of intersections, but not effective for optimization. Another dotted line which falls outside this quadrant is omitted from the figure. 
TABle 2

Multiple regression analysis of two instructional treatment factors and two aptitude informations using immediate test as the dependent variable

\begin{tabular}{clcccccc}
\hline $\begin{array}{c}\text { Step number } \\
\text { and priority } \\
\text { of variable }\end{array}$ & $\begin{array}{c}\text { Variable } \\
\text { entered }\end{array}$ & $\begin{array}{c}\text { Number of } \\
\text { variables } \\
\text { included }\end{array}$ & $\begin{array}{c}\text { Multiple } \\
R\end{array}$ & $\begin{array}{c}\text { Increase } \\
\text { in } R^{2}\end{array}$ & $\begin{array}{c}F \\
\text { ratio }\end{array}$ & $d f$ & $P$ \\
\hline 1 & A B C D & 1 & 0.0721 & 0.0052 & 1.16 & $1 / 222$ & $0.25<P$ \\
2 & A C D & 2 & 0.1976 & 0.0338 & 7.78 & $1 / 221$ & $P<0.01$ \\
3 & B C D & 3 & 0.2026 & 0.0020 & 0.47 & $1 / 220$ & $n s$ \\
4 & A B C & 4 & 0.2157 & 0.0055 & 1.26 & $1 / 219$ & $0.25<P$ \\
5 & A B D & 5 & 0.2158 & 0.0000 & 0.01 & $1 / 218$ & $n s$ \\
6 & A C & 6 & 0.2461 & 0.0140 & 3.24 & $1 / 217$ & $0.05<P<0.10$ \\
7 & B D & 7 & 0.2630 & 0.0086 & 1.99 & $1 / 216$ & $0.10<P<0.25$ \\
8 & B C & 8 & 0.2706 & 0.0041 & 0.94 & $1 / 215$ & $n s$ \\
9 & C D & 9 & 0.2754 & 0.0026 & 0.61 & $1 / 214$ & $n s$ \\
10 & A D & 10 & 0.2754 & 0.0000 & 0.00 & $1 / 213$ & $n s$ \\
11 & A B & 11 & 0.2760 & 0.0003 & 0.08 & $1 / 212$ & $n s$ \\
12 & A & 12 & 0.3286 & 0.0318 & 7.52 & $1 / 211$ & $P<0.01$ \\
13 & B & 13 & 0.3296 & 0.0006 & 0.15 & $1 / 210$ & $n s$ \\
14 & C & 14 & 0.5479 & 0.1916 & 57.24 & $1 / 209$ & $P<0.001$ \\
15 & D & 15 & 0.5485 & 0.0006 & 0.18 & $1 / 208$ & $n s$
\end{tabular}

Factors: A (T-P), B (Num-Gra), C (ISS), D (GAT)

Optimal treatment with ISS and AState is shown in Fig. 3. One of the ATI terms of this case, viz. T-P $\times$ ISS $\times$ AState, using another full model with a priority given to higher-order interaction

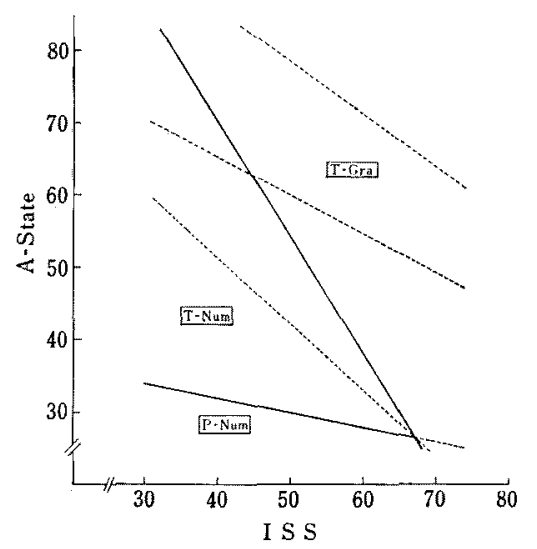

FIG. 3. Regions of optimal treatment when ISS and State-Anxiety are used as aptitude. Dotted lines are also orthogonal projections of intersections, but not effective for optimization. Another dotted line which falls outside this quadrant is omitted from the figure. terms, reached the significance level ( $F$ $=9.25, d f=1 / 221, p<.01)$. P-Gra condition did not appear in both optimal solutions owing to its overall low level in payoff. In addition, payoff dimension in these analyses was immediate test score. Mention will be made of the retention test in another section.

Determination of Optimal Instructional Treatment when Single Aptitude Dimension is Used

Multiple regression analysis resulted in significant ATI effects of T-P $\times$ ISS $(F=$ 21.44, $d f=1 / 277, p<.01)$ and T-P $\times$ GAT $(F=7.58, d f=1 / 221, \quad p<.01)$. In both cases, the priority of ordering was given to interaction terms and reduced models were used as mentioned above. These results partly support the reliability of the following two figures of ATI.

Regression lines of each treatment on ISS are presented in Fig. 4. Disordinal interaction effective for optimization can be obtained between the T-Gra and TNum conditions. Placement to either 
treatment should be done at the crossoverpoint of 50 on the ISS dimension. The authors are of the opinion that assignment to either treatment should be performed just at the crossover-point unless a priority based on some good reason, for example, the problem of cost or difficulty in practice, can legitimately be given to either treatment. The significance region determined by the Johnson-Neyman technique has practical importance only when such a priority is deservedly acknowledged. Taking the risk of Type I error does no harm in the optimization of instructional treatment.

In ATI using GAT as aptitude, the slope of P-Num line is quite different from other treatments and approximates a value of zero (Fig. 5). This treatment is effcient for optimization showing disordinal interaction with the T-Gra treatment and the crossover-point is at 67 on the GAT co-ordinate. Thus, assignment for optimization should be performed using these two treatments.

\section{Main Effects}

Multiple regression analysis resulted in significant main effects of T-P and ISS using the full model with the order-priority given to the interaction terms (Table 2).

As regards the significant main effect of T-P, text format yielded larger mean

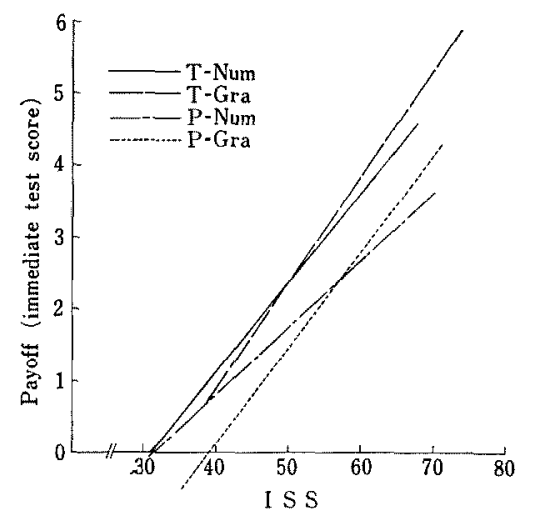

FIg. 4. Regression-lines of four instructional treatments when ISS is used as aptitued. payoff than that of program format (cf. Table 1). When a learning task has a logical and self-contained structure as is the case with the present study, text format is more advantageous than programmed material format which tends to present subject matter in a fragmented way. This result is in agreement with many critical comments on programmed instruction. The payoff of P-Num treatment, however, is least influenced by GAT, as shown in Fig. 5. This fact suggests that program format is more fitting to learners with high anxiety. The effectiveness of instructional method should be evaluated, not from the view point of main effect alone, but from that of ATI.

\section{Comparison between Payoffs of Four Treatment} Conditions and Optimized Treatments

Means and standard deviations of payoffs in four instructional treatments and optimized treatments are presented in Tables 1 and 3 respectively. As for optimized treatments, the following three means are calculated, using both ISS and GAT as aptitude information, ISS alone, and GAT alone. In addition, three methods of calculation were devised. The first one, named "Optimally instructed" in Table 3, picked up every subject who happened to be treated under the optimal condition for him. For example, using

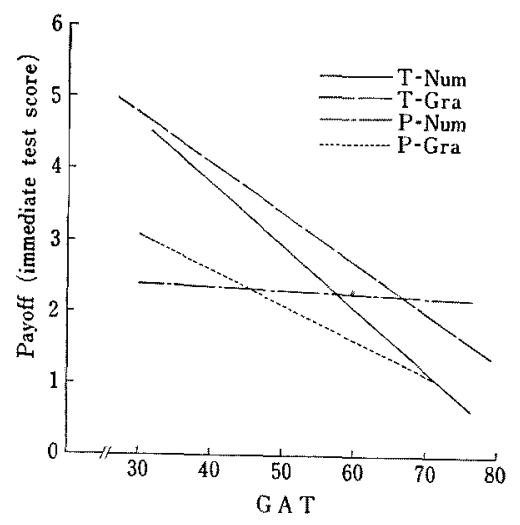

FIG. 5. Regression-lines of four instructional treatments when GAT is used as aptitude. 
TABLE 3

Comparison of optimized treatments using two or single aptitude information in terms of three kinds of mean payoffs

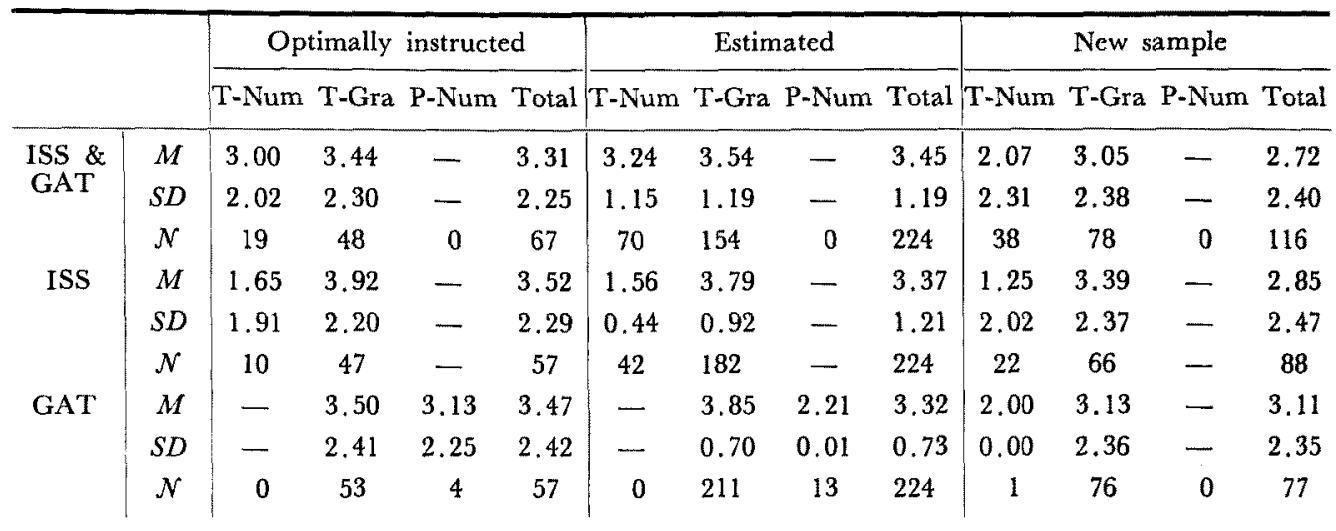

ISS and GAT as aptitude, 19 subjects chanced to be treated under their optimal condition of T-Num, 48 under T-Gra, and none under P-Num (P-Gra did not appear as an optimal condition at all). In total, 67 subjects were optimally treated, yielding a mean payoff of 3.31 . The second method, named "Estimated", assigned every subject to his optimal condition in terms of his aptitudes, and his payoff was estimated by means of regression planes or lines. Mention will be made of the last method, "New sample", in the next section.

The measure "Estimated" shows an overall higher value and smaller standard deviation because it represents an ideally optimized payoff on the regression plane or line switched over to a better payoff. Thus, the first measure provides a more conservative estimation of mean payoff than the second one.

Mean payoffs tend surely to be improved by optimization. The improvement, however, is small in quantity, and this fact may be interpreted in terms of the high generality of both ISS and GAT, as Cronbach (1967) persuasively points out.

Special attention should be paid to the fact that the improvement in mean payoff yielded by optimization is larger when a single aptitude is considered than when two are taken into account. In so far as payoff alone is regarded as the criterion, single aptitude information suffices, and taking two aptitude informations into consideration is not so much redundant as disadvantageous. However, from the standpoint of adapting instructional treatment to individual differences, two or more aptitude informations might be recommended as suitable even at the cost of a small loss in payoff. The alternatives are yet open to future research.

Application of the Obtained Method of Optimization to a New Sample of Subjects

To check the stability and practical utility of the optimizing method thus obtained, a new sample of 116 subjects was assigned to each optimal treatment in terms of Figs. 2, 4 and 5. Since this application experiment was conducted on pupils of the same grade from the same school in the same month of the next year, the scholastic aptitude level of the new sample could be assumed to be approximately equal to that of the original sample. First, based on ISS and GAT scores measured in advance, each subject was assigned to his optimal treatment in Fig. 2. The resulting number of subjects assigned to each treatment, the mean payoff for 
each, and the total mean of optimized payoff are shown in Table 3 . There were no subjects of this new sample assigned to P-Gra treatment, as observed in the original sample. As the next step, payoff scores obtained from optimization using ISS and GAT were regrouped, respectively, in terms of ISS alone (Fig. 4) and GAT alone (Fig. 5). These results are also presented in Table 3. The total mean payoff in the latter case (3.11) is likely to be a higher estimation because there were no subjects assigned to P-Num treatment after regrouping of the payoff scores obtained from optimization using ISS and GAT.

Inspection of these means suggests that optimization using two aptitude informations tends to yield a lower payoff than when a single aptitude is used, as pointed out in the last section. The overall decline of payoffs in the new sample may be ascribed to the attenuation caused by applying the result of multiple regression obtained from the original sample to the new sample. In addition, the decrement might be interpreted as the result of the subtle difference in the instruction given to subjects. In the original experiment, subjects were informed beforehand of the test which was to be administered after the learning session; several cases of cheating in tests were detected. Consequently, in the application experiment, subjects were not informed of the test in advance. This difference, together with the above attenuation, might have caused the decrement in payoff.

\section{Retention Test Score}

All of the payoff dimensions hitherto mentioned are the scores of the immediate test. An example of ATI using the retention test score as payoff is shown in Fig. 6. The aptitude information is ISS and the interaction presents a similar pattern to that of the immediate test score except that overall payoff is lower (cf. Fig. 4). Multiple regression analysis proved that the contribution of ISS was markedly larger in comparison with that of other variables. The reason might be that items of the retention test were identical to those of the immediate test, and favorable to subjects with high general intelligence.

With respect to the means of the four instructional treatments, T-Gra condition was the highest; the order of magnitude was the same as that of the immediate test score (Table 1).

Generality vs. Specificity in Aptitude Information

In the present experiment, pretest score had been selected as a variable with high specificity, general intelligence as one with high generality, and grade point of mathematics as one lying between these two. Pretest score had very low correlation with payoff, partly because its difficulty level was too low for the subjects and it showed a ceiling effect. Thus, no disordinal interaction efficient for optimization was obtained. Nor did the grade point of mathematics show any ATI effect. Eventually, only general intelligence resulted in significant ATI effects as mentioned above.

With regard to the emotional aspect, A-State score had been taken as a variable with high specificity and GAT, as one with high generality. Again, in this

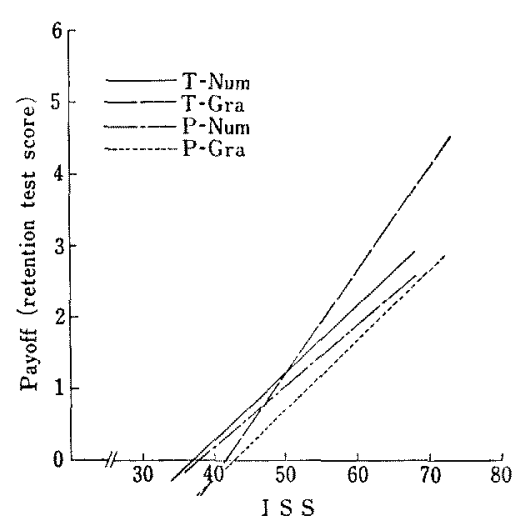

FIG. 6. Regression-lines of four instructional treatments using ISS as aptitude and retention test score as payoff. 
case, significant ATIs were observed only in GAT, with the exception of the triple interaction of T-P $\times$ ISS $\times$ A-State. The fact that T-P $\times$ A-State does not reach the significance level suggests that the significant triple interaction results only when A-State is combined with ISS.

So far as results of the present study are concerned, the general aptitude information has an advantage over the specific in yielding a significant ATI. These results are in perfect agreement with Cronbach's recent conclusion (1975). The senior author of this paper has a further discussion of this problem elsewhere (Namiki, 1977).

\section{Conclusions}

Contrary to expectation, increasing the number of aptitude informations in optimization did not produce a higher payoff. In so far as a payoff measure of this sort is regarded as the criterion, single aptitude information suffices. The question is whether or not the measure of payoff has pertinence to our notions about learning outcomes. This consideration suggests that the measure of payoff should be much more multidimensional than that of the present study, as Cronbach (1966) has persuasively pointed out.

Among ATIs with single aptitude information, GAT yielded relatively conspicuous disordinal interaction which was considered valid for optimization. This finding indicates an interesting aspect of programmed textbooks, one which deserves further research.

On the whole, the improvement of payoff brought about by optimization is too small in quantity. The extrapolation from the present experiment to the practical setting is not large; therefore, it is the authors' hope that the evidence thus obtained from the present experiment, even if small in quantity, has relevance to practice. The practical utility of this evidence, however, must be examined in terms of a different view point apart from that of the present experiment.

\section{REFERENGES}

Azuma, H. 1968 Optimization of instructional guidance. In Hatano, et al. (Eds.) Handbook of psychology of learning $\mathcal{G}^{\circ}$ instruction. Tokyo: Kanekoshobo, Pp 633-647. (In Japanese)

Cohen, J. 1968 Multiple regression as a general data analytic system. Psychological Bulletin, 70, 426-443.

Cronbach, L.J. 1966 The logic of experiments on discovery. In L.S. Shulman \& E. R. Keislar (Eds.), Learning by discovery: A critical appraisal. Chicago: Rand McNally \& Co., Pp 77-92.

Gronbach, L.J. 1967 How can instruction be adapted to individual difference? In R. M. Gagné (Ed.), Learning and individual differences. Columbus: Charles E. Merrill Publishing Co., Pp 23-39.

Cronbach, L.J. 1975 Beyond the two disciplines of scientific psychology. American Psychologist, 30, 116-127.

Cronbach, L.J., \& Snow, R. E. 1969 Individual differences in learning ability as a function of instructional variables. (U.S. Office of Education Report 4-6-061269-1217), Stanford, Calif.: Stanford Univ.

Gagné, R. M., \& Brown, I. 'T. 1961 Some factors in the programming of conceptual learning. Fournal of Experimental Psychology, 62, 313-321.

Hayashi, J., \& Namiki, H. 1975 The ATI effect in learning of solution rules. Proceedings of 17th Annual Convention of the Japanese Association of Educational Psychology, 424-425. (In Japanese)

Kerlinger, F. N., \& Pedhazur, E.J. 1973 Multiple regression in behavioral research. N.Y.: Holt, Rinehart and Winston, Inc.

Koran, M. L., Snow, R. E., \& MaDonald, F. J. 1971 Teacher aptitude and observational learning of a teaching skill. Fournal of Educational Psychology, 62, 219-228.

NAMIKI, H. 1977 Specificity-generality in ATI research: Some theoretical consideration and experimental evidences. In Collection of papers dedicated to Keizo Hayashi, 9th report, Psychological Laboratory on the Hiyoshi Campus, Keio Univ., 87-98.

Namiki, H., Hayashi, J., \& Kazama, N. 1977 
The ATI effect in problem solving learning: When two aptitude informations are used. Studies in Sociology, Psychology and Education, Keio Univ., 17, 1-9. (In Japanese)

Overall, J. E., \& Spiegel, D. K. 1969 Concerning least squares analysis of experimental data. Psychological Bulletin, 72, 311-322.

PotтhofF, R. F. 1964 On the Johnson-Neyman technique and some extensions thereof. Psychometrika, 29, 241-256.

Rhetrs, J. E. 1974 Task, learner, and treat- ment variables in instructional design. Journal of Educational Psychology, 66, 339-347.

Spielberger, C. D. 1966 The effect of anxiety on complex learning and academic achievement. In C. D. Spielberger (Ed.), Anxiety and behavior. N.Y.: Academic Press.

Tobias, S. 1973 Sequence, familiarity, and attribute by treatment interactions in programmed instruction. Fournal of Educational Psychology, 64, 133-141.

(Received Feb. 18, 1977) 